\title{
Nano/sub-nano analysis based on high resolution transmission electron microscopy for ceramic materials
}

\author{
Takahisa YAMAMOTO ${ }^{\dagger}$ \\ Department of Advanced Materials Science, The University of Tokyo, 5-1-5 Kashiwanoha, Kashiwa, Chiba 277-8561
}

Grain boundaries, interfaces and surfaces are very attractive areas from the viewpoint of lattice discontinuity. Doped elements and point defects often segregate there, which largely changes bulk properties such as microstructure, mechanical strength, electrical conductivity. To understand the details of the dopant effects, it is necessary to use nano-scale analysis techniques based on transmission electron microscopy with energy dispersive $X$-ray spectroscopy and electron energy-loss spectroscopy with nano/sub-nano electron probe. This review contains some nano-scale analysis studies performed in our research group for the grain size control of $\mathrm{BaTiO}_{3}$ polycrystals, electrical properties across single grain boundaries of n-type $\mathrm{BaTiO}_{3}$, $\mathrm{SrTiO}_{3}$ and $\mathrm{ZnO}$, interface structural change of WC-Co based cemented carbides and the preparation of novel dislocation nano-wires.

Grain boundaries in $\mathrm{BaTiO}_{3}$ sinters were revealed to exhibit grain boundary faceting by doping a very small amount of excess $\mathrm{TiO}_{2}$. The formation of the facets due to extra $\mathrm{Ti}-\mathrm{O}_{2}$ bonding is closely related to abnormal grain growth, which is often observed in $\mathrm{TiO}_{2}$-excess $\mathrm{BaTiO}_{3}$ sinters. A similar faceting feature is also observed in VC-doped WC-Co cemented carbides. Doped VC strongly segregates to $\mathrm{WC} / \mathrm{Co}$ interfaces to form micro facets. The formation of the micro facets resulted in WC grain size reduction. In $\mathrm{ZnO}$ boundaries, doped Pr was revealed to segregate at the grain boundary. The segregated Pr ions are situated in specific atomic columns. Further, electron energy-loss spectroscopy has revealed that the valence of the segregated Pr ions is $3+$, which means that they are not acceptors but donors to $\mathrm{ZnO}$. The improvement of varistic effect observed in Pr-doped $\mathrm{ZnO}$ is closely related to $\mathrm{Zn}$ vacancies enhanced by $\mathrm{Pr}$ doping. Meanwhile, dislocations are a kind of one-dimensional lattice defect including extra half planes. By pipe-diffusion of dopants at dislocation cores, we can successfully develop conducting nano-wires in insulating sapphire crystals.

(C2011 The Ceramic Society of Japan. All rights reserved.

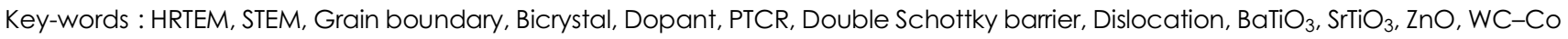

[Received July 28, 2010; Accepted December 16, 2010]

\section{Introduction}

Crystalline defects such as grain boundaries, interfaces, dislocations and point defects are source of many interesting properties which are not normally found in perfect crystalline structures. ${ }^{1) 2}$ It has been recognized that the segregation of dopants to these defects often dramatically changes their properties and thus controls a whole property of bulk materials. For example, very small amount of Pr doping, which segregate to grain boundaries, are known to be very effective to improve varistic properties of n-type $\mathrm{ZnO}$-based ceramics. ${ }^{3), 4)}$ In WC-Co based cemented carbides, $\mathrm{VC}$ is known to be an effective inhibitor for WC grain growth behaviors. ${ }^{5)-7)}$ Positive temperature coefficient of resistivity (PTCR) effect in n-type $\mathrm{BaTiO}_{3}$ based sinters is improved by Mn doping to grain boundaries. ${ }^{8)-11)}$ Rare earth dopants are well known to increase the sinterbility of $\mathrm{Si}_{3} \mathrm{~N}_{4}{ }^{12)}$ and creep resistance of $\mathrm{Al}_{2} \mathrm{O}_{3}$ ceramics by segregating to grain boundaries. ${ }^{13), 14)}$ While we can expect these dopants modify the properties of defects, it is essential to analyze the detailed structures of dopants at the nano-scale defects in order to understand the precise mechanism of dopant effect. The technique based on transmission electron microscopy (TEM) is one of the most powerful techniques for this purpose. ${ }^{15)-18)}$ TEM basically utilize round type magnetic lenses to form magnified

Corresponding author: T. Yamamoto; E-mail: yamataka@ k.u-tokyo.ac.jp image. Initially, the obtained resolution were limited by the lens system, but easy operation even at an atomic resolution level now becomes possible with the improvement of magnetic lens and the stability of whole microscope system. In addition, in recent days, aberration correction system with multiple pole type lenses has been developed and now become commercially available. ${ }^{19)} \mathrm{A}$ round type lens is a convex lens with inevitable positive spherical aberration which has limited a resolution and an electron probe size. In principle, the spherical aberration cannot be compensated by a lens system with only round type lenses. ${ }^{20)}$ However, the combination of multiple pole type lenses can serve as a concave lens with negative spherical aberration. By systemizing the pole type lenses to the microscope column, what we call Cs-corrector, spherical aberration in the lens system can be effectively corrected. To set a Cs-corrector above an objective lens, very sharp electron probe less than $0.1 \mathrm{~nm}$ can be now achieved. As a result, ultra high resolution scanning transmission electron microscopy (STEM) has become in practical use. ${ }^{21)}$ In addition to the improvement in resolution, there are several merits in STEM. By controlling detection angles of transmitted and/or scattered electrons with annular type detectors, the following imaging becomes possible; direct atomic column imaging, atomic number $(Z)$ dependent contrast imaging, ${ }^{22)}$ strain field sensitive imaging and so on. STEM now enables us to directly characterize detailed structures of crystalline defects at atomic dimensions including the detection of electronic states at single atomic column by electron energy loss spectroscopy. ${ }^{23)}$ 
Several researchers have been extensively studying dopant effects at crystalline defects using TEM, high resolution TEM and STEM. In ZnO-based varistors, doped Pr has been revealed to be a donor by direct analysis of segregated Pr ions with EELS analysis. ${ }^{24)}$ In $\mathrm{WC}-\mathrm{Co}$ based cemented carbides, doped VC forms $(\mathrm{W}, \mathrm{V}) \mathrm{C}_{X}$ compounds as layers on a surface of WC grains that strongly retards the step growth of the surface. ${ }^{7), 25), 26)}$ Rareearth ions doped in $\mathrm{Si}_{3} \mathrm{~N}_{4}$ forms strong bonding to the particular surfaces of $\mathrm{Si}_{3} \mathrm{~N}_{4}$ grains. ${ }^{27), 28)}$ In rare-earth doped $\mathrm{Al}_{2} \mathrm{O}_{3}$, doped rare earth ions occupy specific atomic positions at grain boundaries to strengthen them by forming strong covalent-like bonds in their vicinity. ${ }^{29)}$ These results suggest that controlling dopant segregation to the crystalline defects may lead to the new way of developing materials and devices from the atomic-scale.

Our research group has studied the structures and properties of crystalline defects in various kinds of ceramic materials by TEM and STEM. In this paper, we review some key topics such as grain size control of $\mathrm{BaTiO}_{3}$ polycrystals, electrical properties across single grain boundaries of n-type $\mathrm{BaTiO}_{3}, \mathrm{SrTiO}_{3}$ and $\mathrm{ZnO}$, interface structural change of $\mathrm{WC}-\mathrm{Co}$ based cemented carbides. In addition, we present a novel nano-wire fabrication method using dislocations as a nano-scale template for dopant atoms.

\section{Control of grain growth behavior in $\mathrm{BaTiO}_{3}$}

$\mathrm{BaTiO}_{3}$ (BTO) polycrystals are often prepared by liquidphase sintering with excess $\mathrm{TiO}_{2}$ and $\mathrm{SiO}_{2}$ as a sintering aid. ${ }^{30}$ ) During the liquid-phase sintering, some grains often grow rapidly, which is called abnormal grain growth (AGG). The AGG is triggered mostly by inhomogeneous distribution of thin liquid film formed at grain boundaries. However, AGG is also observed in solid-phase sintering below eutectic temperature between $\mathrm{BTO}$ and $\mathrm{TiO}_{2} \cdot{ }^{31-35)}$ As for the AGG below the eutectic temperature, the twin growth mechanism has been proposed, which is proposed from a fact that most of abnormal grains include $\{111\}$ twins. $^{36}$ ) The $\{111\}$ twin grows with forming reentrant edges including excess Ti. The abnormal grains exhibit elongated shapes parallel to $\{111\}$ twins. In contrast, Kang's group investigated the details for the AGG from the viewpoint of grain boundary structure transition. ${ }^{37), 38)}$ They reported that the AGG is very sensitive to oxygen partial pressure during sintering. Even if $\{111\}$ twins are included in grains, the grains never exhibit AGG in oxidizing atmosphere. The phenomenon is closely related to grain boundary structure transition induced by variation of oxygen partial pressure. Grain boundaries become atomically rough in reducing atmosphere while singular (flat) in oxidizing atmosphere. The rough/ singular structure change due to variation of oxygen partial pressure is called as defacet/facet transition. The important point is that the AGG starts from a singular boundary distributed in rough boundaries. Grains whose growth is strongly suppressed rapidly grow at a critical driving force. In recent, a simulation showing AGG is reported. ${ }^{39)}$ The simulation clearly showed the importance of singular boundary for AGG. ${ }^{39)}$ In Ti-excess $\mathrm{BaTiO}_{3}$ (BTO), a clear singular boundary is formed while Ba-excess BTO exhibits rough boundaries. ${ }^{40)}$ It is suggesting that the AGG is very sensitive to a small shift from the stoichiometric ratio of cations in a perovskite structure. Figure 1 shows optical micrographs taken from (a) Ti-excess BTO and (b) Ba-excess BTO, which are sintered by solid-phase sintering. ${ }^{41)}$ The $\mathrm{Ba} / \mathrm{Ti}$ ratio change of the respective sinters is very small at a level of $1 / 1000$. As shown in the two optical micrographs, Ti-excess BTO exhibits AGG while Ba-excess

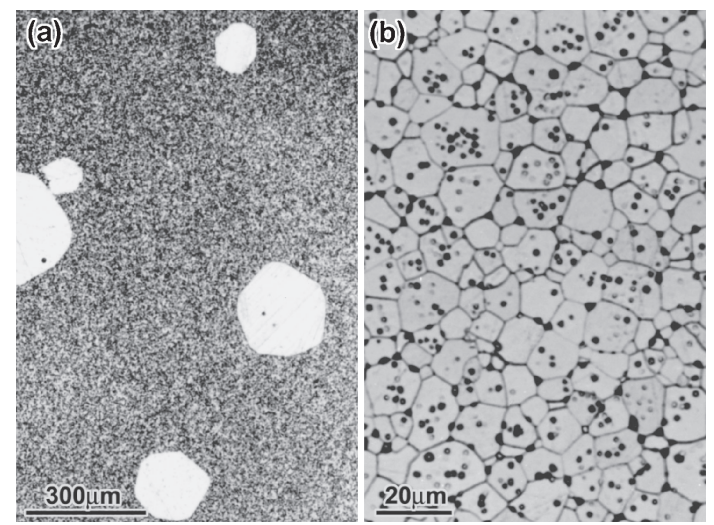

Fig. 1. Optical micrographs of (a) Ti-excess and (b) Ba-excess $\mathrm{BaTiO}_{3}$ $\mathrm{The} \mathrm{Ba} / \mathrm{Ti}$ raito of the respective sinters are 0.999 in (a) and 1.001 in (b).

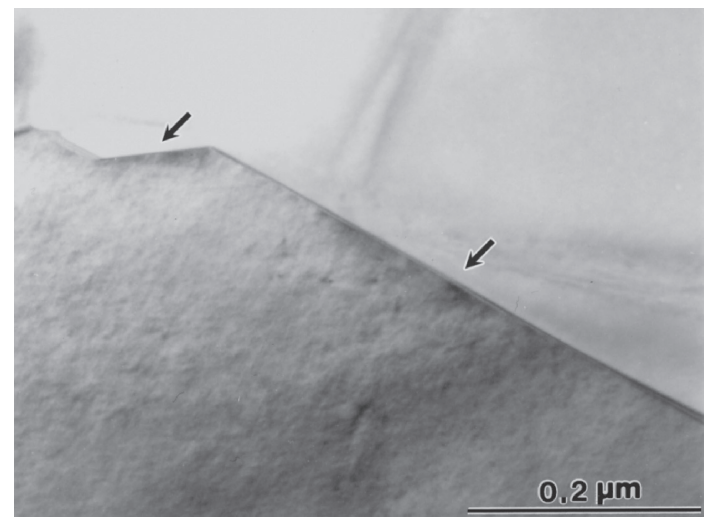

Fig. 2. TEM bright field image at a facetted boundary in Ti-excess $\mathrm{BaTiO}_{3}$. The habits at the facet boundaries are mostly $\{210\}$ types.

BTO shows a uniform grained structure (normal grain growth, NGG). The difference in the grain growth behavior observed in BTO sinters is closely related to a grain boundary structural change induced by a small shift of $\mathrm{Ba} / \mathrm{Ti}$ ratio. Transmission electron microscopy (TEM) analysis for the grain boundaries revealed that Ti-excess BTO exhibits faceted grain boundaries. Figure 2 shows a typical example of the facetted boundary formed in Ti-excess BTO. ${ }^{41)}$ As seen in the figure, the grain boundary exhibits clear faceting features mostly consisting of $\{210\}$ habit planes. The important point is that such habits are never observed in Ba-excess BTO that shows NGG. This means that NGG/AGG transition is closely related to the appearance of faceted boundaries. $\{210\}$ in a perovskite structure has a stacking sequence of $\mathrm{SrO}$ and $\mathrm{TiO}_{2}$ planes. As the facets are observed only in Ti-excess BTO, the terminated planes at the habits must be $\mathrm{TiO}_{2}$ planes. At a high resolution level, an interesting structure can be seen at the habit as shown in Fig. 3. ${ }^{41)}$ In the image, the habit plane is oriented parallel to the electron beam. The grain on the right hand side is set at a zone axis of (001) while the other is not. As seen along the habit plane, particular contrast can be seen periodically as indicated with arrows, which means the formation of an extra structure on the habit plane. By taking EELS, the particular structure was found to have an extra $\mathrm{TiO}_{2}$ bonding. Figures 4(a) and 4(b) show oxygen K-edge ELNES, which are taken from the areas at a grain interior and at a habit plane of Ti-excess BTO. ${ }^{41)}$ Theoretical ELNES is also shown for comparison in Figs. 4(c) and 4(d). In the experimental ELNES from the grain interior (a), three main peaks appear as expected from the theoretical 


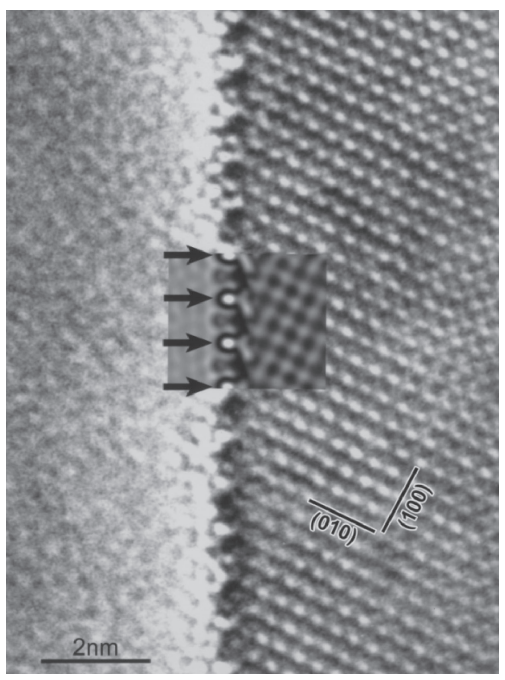

Fig. 3. HRTEM image taken from a habit plane. In the image, the grain on the right hand side is set at (001). It is noted that periodic bright contrasts can be seen along the habit plane. At the center of the image, a simulated image is superimposed, which was constructed by an atomic model including extra $\mathrm{TiO}_{2}$ bonding.

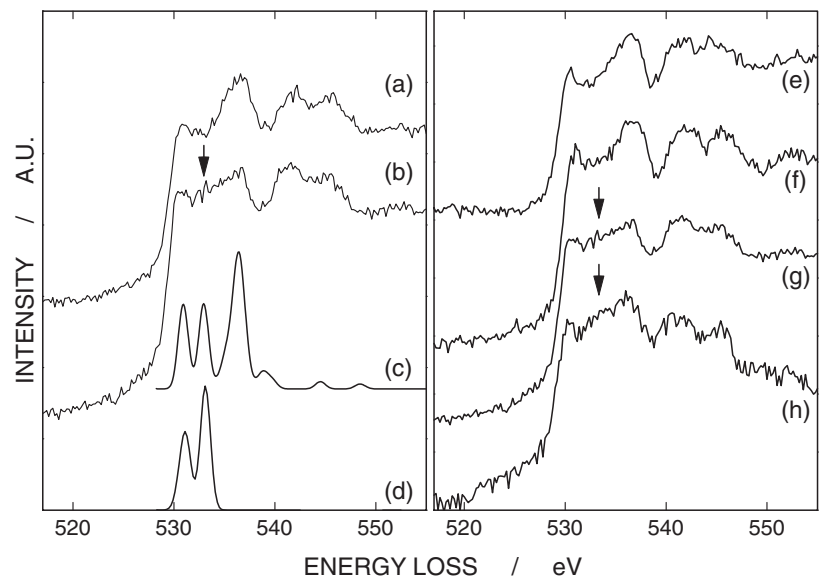

Fig. 4. Oxygen K-edge ELNES. (a) and (b) a grain interior and a facetted boundary of Ti-excess $\mathrm{BaTiO}_{3}$. (c) and (d) theoretical profiles of $\mathrm{BaTiO}_{3}$ and of $\mathrm{TiO}_{2}$. (e), (f), (g) and (h) grain boundaries of $\mathrm{Y}, \mathrm{Ca}, \mathrm{Nb}$ and $\mathrm{Zr}$ doped $\mathrm{BaTiO}_{3}$. Y and $\mathrm{Ca}$ doped $\mathrm{BaTiO}_{3}$ exhibit $\mathrm{NGG}$ and $\mathrm{Nb}$ and $\mathrm{Zr}$ doped $\mathrm{BaTiO}_{3}$ exhibit AGG.

ELNES as shown in (c). On the other hand, the facetted boundary shows the plateau-like profile as indicated by an arrow. This plateau feature arises from the overlapping of the peaks from $\mathrm{BaTiO}_{3}$ and $\mathrm{TiO}_{2}$. If the $\mathrm{TiO}_{2}$ rutile structure is formed at the facetted boundary, the EELS will reflect those from $\mathrm{TiO}_{2}$ and $\mathrm{BaTiO}_{3}$. Both of the perovskite and rutile structures have $\mathrm{TiO}_{6}$ octahedrons, but the linkage of the $\mathrm{TiO}_{6}$ octahedrons is different between the two structures. $\mathrm{TiO}_{6}$ octahedron has a corner-shared linkage in perovskite structure while rutile structure includes the edge-shared $\mathrm{TiO}_{6}$ octahedrons. Therefore, the bonding angle of $\mathrm{Ti}-\mathrm{O}-\mathrm{Ti}$ is $180^{\circ}$ in perovskite structure and about $100^{\circ}$ in rutile. ${ }^{42)}$ As a result, the pre-peak (a first peak) of the O K-edge has higher intensity than the second peak in the perovskite structure [Fig. 4(c)] while the intensity of the second peak is lower in the rutile structure [Fig. 4(d)]. As seen in the simulated image superimposed in the experimental HRTEM image of Fig. 3, a model including the extra $\mathrm{TiO}_{2}$ bonding agrees well with the periodic contrast.
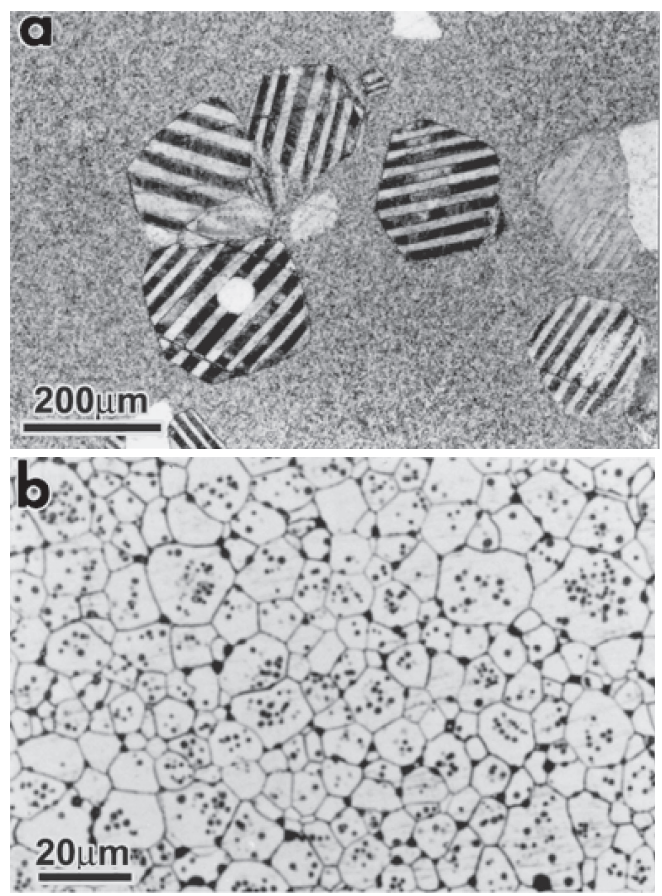

Fig. 5. Optical micrographs of (a) $0.1 \mathrm{Y}-0.2 \mathrm{Ti}$ and (b) $0.1 \mathrm{Nb}-0.2 \mathrm{Ba}$ doped $\mathrm{BaTiO}_{3}$

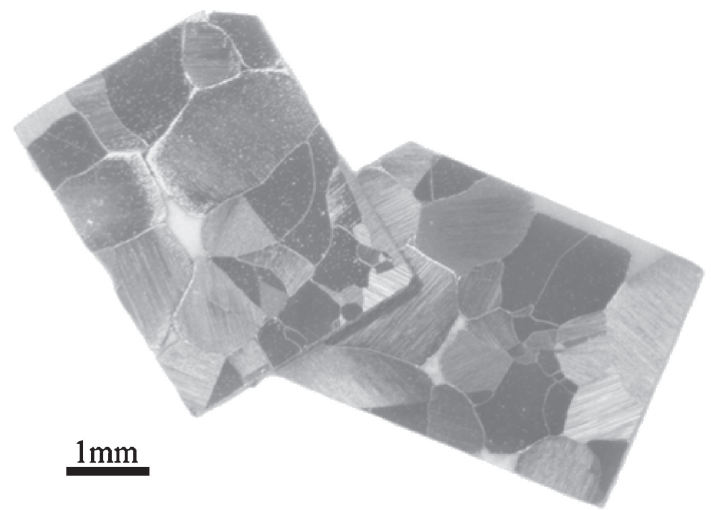

Fig. 6. An optical photograph of coarse grained $\mathrm{BaTiO}_{3}$.

The sensitivity of AGG to the off-stoichiometric $\mathrm{Ba} / \mathrm{Ti}$ ratio is similarly observed in doped BTO. ${ }^{43}$ The grain growth in doped BTO also exhibits the sensitivity to a small shift of A/B-site ratio considering the doped cation's substituting sites in a perovskite structure. A typical example is shown in Fig. 5. ${ }^{43)}$ The two optical micrographs show etched sections of $0.1 \mathrm{Y}-0.2 \mathrm{Ti}$ and $0.1 \mathrm{Nb}-0.2 \mathrm{Ba}$ doped $\mathrm{BaTiO}_{3}$. A dopant of $\mathrm{Y}$ substitutes at the A-site and that of $\mathrm{Nb}$ at the B-site in a perovskite structure from the viewpoint of their ionic radius. So, the total A/B-site ratio in the respective sinters is $\mathrm{B}$-site excess and A-site excess. In the case that $\mathrm{Y}$ and $\mathrm{Nb}$ are solely doped to $\mathrm{BTO}$, the respective sinters exhibit NGG and AGG. In contrast, $0.1 \mathrm{Y}-0.2 \mathrm{Ti}$ doped BTO shows AGG and $0.1 \mathrm{Nb}-0.2 \mathrm{Ba}$ doped BTO NGG by doping additional $\mathrm{Ti}$ and $\mathrm{Ba}$, respectively. In doped $\mathrm{BTO}$, a change in the O K-edge ELNES can also be observed as shown in Figs. 4(e), 4(f), 4(g) and 4(h). Plateau feature in O K-edge ELNES can be seen at grain boundaries also in doped BTO that shows AGG. ${ }^{44)}$

By using the AGG/NGG transition due to $\mathrm{A} / \mathrm{B}$ ratio change, we can prepare unique BTO sinters. Figure 6 is an optical micrograph of BTO sinters prepared by precisely controlling 
A/B ratio. ${ }^{45)}$ Grains around $1 \mathrm{~mm}$ could be obtained even by an ordinary sintering condition. With this type of coarse grained sinters, it becomes possible to fabricate BTO bicrystals by machining. Further, if we put a BTO seed crystal on a BTO polycrystal with a tuned A/B ratio, the single crystal growth can be enhanced from the seed crystal into the polycrystal, and finally large BTO single crystals can be obtained by sintering condition. ${ }^{46)}$

\section{Electron transport behaviors across single grain boundaries}

n-Type BTO polycrystals exhibit positive temperature coefficient of resistivity (PTCR) effect, which is characterized by a large increase of resistivity against temperature. ${ }^{8), 9)}$ PTCR effect is due to double Schottky barriers (DSB) formed at grain boundaries, ${ }^{47)}$ so the PTCR effect can be considered to depend on grain orientation relationships of grain boundaries. ${ }^{45)}$ To develop detailed understanding of the phenomena, a direct measurement of resistance-temperature $(R-T)$ properties across single grain boundaries is necessary. Several groups have performed the direct measurement of PTCR effect at single grain boundaries. ${ }^{45)-52)}$ For example, Nemoto et al. carried out by using fine conducting wires welded at grain interiors. They showed that PTCR effect varies with grain boundaries. Kuwabara et al. used a unique BTO wires. ${ }^{52)}$ They initially cut BTO green compacts into a wire shape and they sintered at higher temperature to induce abnormal grain growth. As a results, they successfully prepared BTO wires including boundaries with the wire width. According to their results, a shape of $R-T$ curves at single boundaries can be separated into three groups, i.e., platue shape, normally shape and saw tooth types. In contrast, we initially prepared coarse grained BTO sinters obtained by controlling AGG as shown in Fig. $6 .{ }^{45}$ ) A size of the BTO sinters is large enough to cut BTO bicrystals. Figure 7 shows $R-T$ curves obtained from various types of single grain boundaries. ${ }^{45}$ ) The resistivity jump observed around the ferroelectric transition point varies with grain boundaries. The resistance jump becomes remarkable at the boundaries with lower coherency while it tends to become smaller or to disappear at boundaries with higher coherency. Namely, PTCR effect at grain boundaries can be discussed in terms of coherency at grain boundaries.

To precisely control grain boundary coherency, it is necessary to tune both the grain boundary plane and the grain orientation relationship. For this purpose, bicrystal experiments using single crystals are more useful. ${ }^{53)-56}$ Figure 8 shows a series of HRTEM images taken from $\mathrm{SrTiO}_{3}$ (STO) bicrystals having various grain orientation relationships. ${ }^{57)}$ As we can see, the grain boundary atomic structures vary with rotational relations

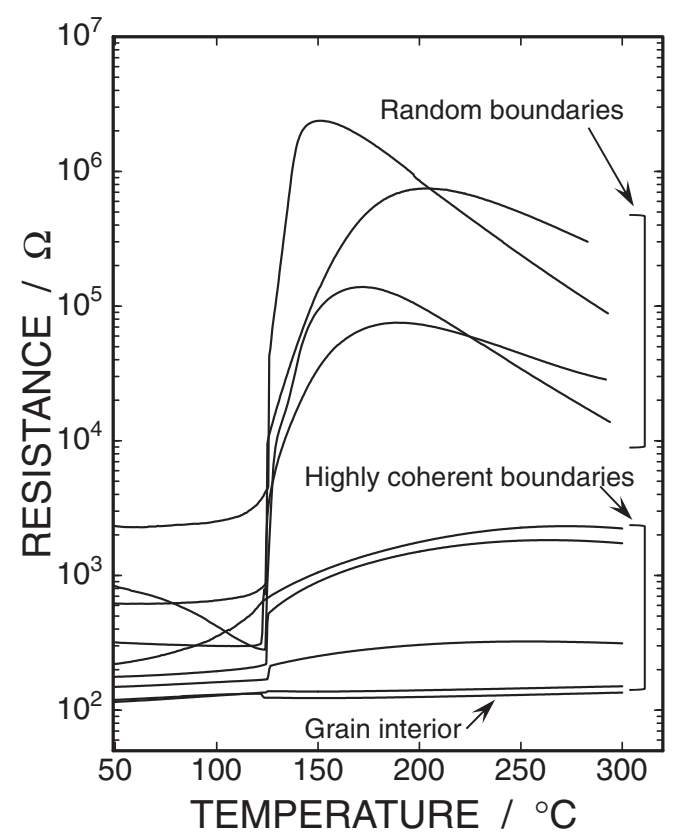

Fig. 7. Resistance-temperature curves obtained from various types of single grain boundaries.

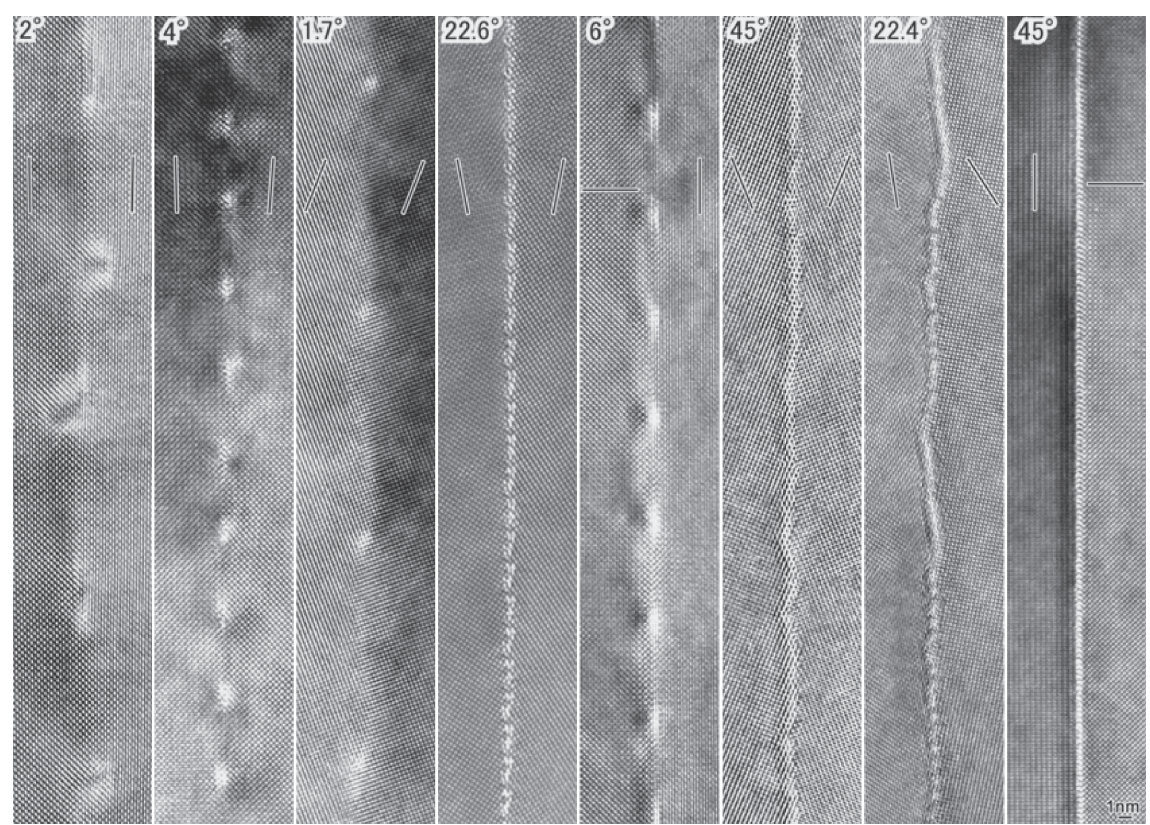

Fig. 8. HRTEM images of $\mathrm{SrTiO}_{3}$ bicrystals prepared by machining, polishing and joing $\mathrm{STO}$ single crystals. In the images, angles indicated at the top of the respective images show the tilt angles and bars show the (010) directions. A rotational axis is [001]. 

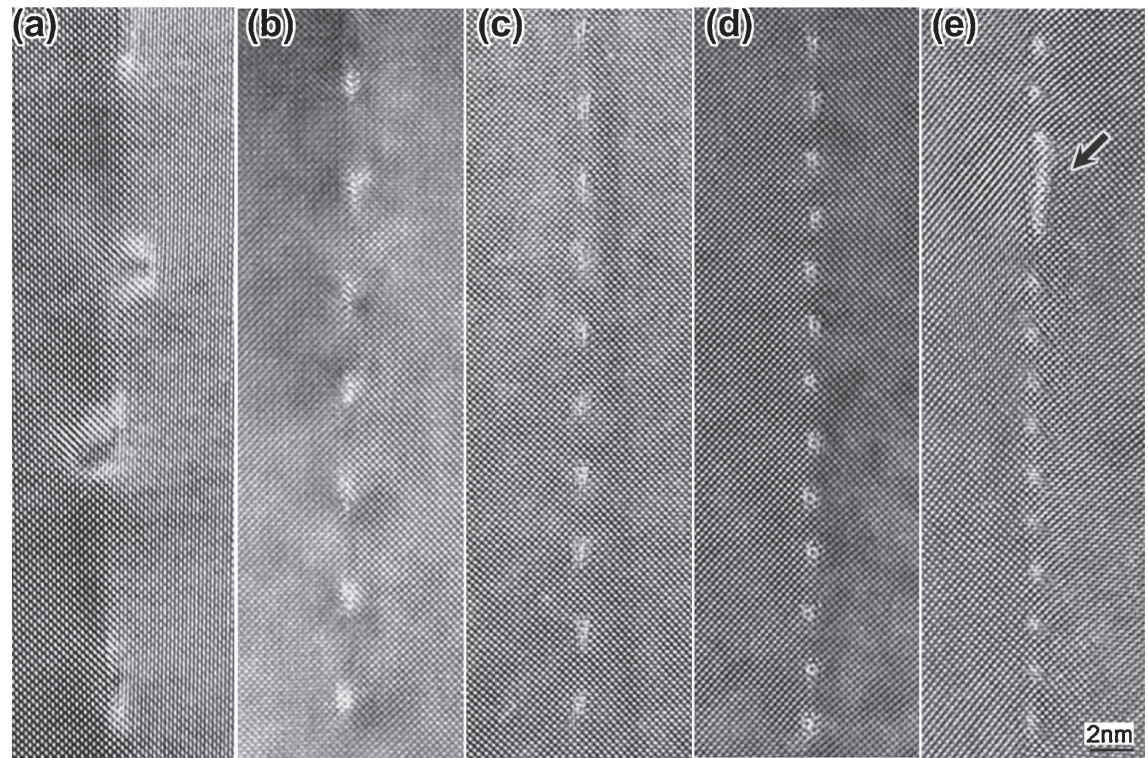

Fig. 9. HRTEM images of low angle boundaries in STO bicrystals. Tilt angles are (a) $2^{\circ}$, (b) $4^{\circ}$, (c) $6^{\circ}$, (d) $8^{\circ}$ and (e) $10^{\circ}$.

between adjacent crystals. In a low angle region, grain boundaries consist of grain boundary dislocations, which are introduced to compensate a small misorientation angle. ${ }^{58)}$ At a special relation, unit structures appear periodically along a grain boundary, which is a coincident site lattice boundary. ${ }^{59)}$ The grain boundary structural change due to a change in rotational relation has a large influence on grain boundary phenomena. For example, n-type STO shows non-linear current-voltage relation due to DSB. The barriers are formed by excess charges mainly given by an inequilibrium distribution of point defects around grain boundaries. Figure 9 shows HRTEM images of low angle boundaries whose tilt angles range from 2 to $10^{\circ}{ }^{58)}$ In the images, white contrast can be seen along the boundaries, showing the positions of grain boundary dislocations. As we can see, the density of grain boundary dislocations increases with tilt angles. However, at the $10^{\circ}$ boundary, another structure appears as shown by an arrow. This means that Brandon's criterion, defined as an angle of overlapping adjacent dislocation cores, exists around $10^{\circ}$ in the case of STO. ${ }^{60)}$ Over the angle, the grain boundary structure transforms from a dislocation structure to a random one. By using a series of the low angle boundaries, we can estimate excess charges given by one dislocation with a unit length. The result is shown in Fig. 10. By taking nonlinearity from the three kinds of low angle boundaries, excess charges necessary to form DSB can be obtained as a function of dislocation numbers as shown in the plot of Fig. 10. One grain boundary dislocation with $1 \mathrm{~nm}$ gives approximately 0.7 electrons. The excess charges are closely related to the inequilibrium distribution of point defects. According to $a b$ initio calculations performed for STO crystals, point defect formation energy of $\mathrm{Sr}$ cations is lowest compared to that of $\mathrm{Ti}$ cations. ${ }^{61)}$ Thus, a distribution of charged $\mathrm{Sr}$ vacancies is a key to induce DSB.

\section{Direct visualization of dopants at a grain boundary}

$\mathrm{ZnO}$ is known to be a typical varistic material. ${ }^{3), 62), 63)}$ In practical use, effective dopants such as $\operatorname{Pr}$ and Bi are often doped to increase the varistic effect. From a viewpoint of DSB properties, such dopants had been thought to be of acceptor type

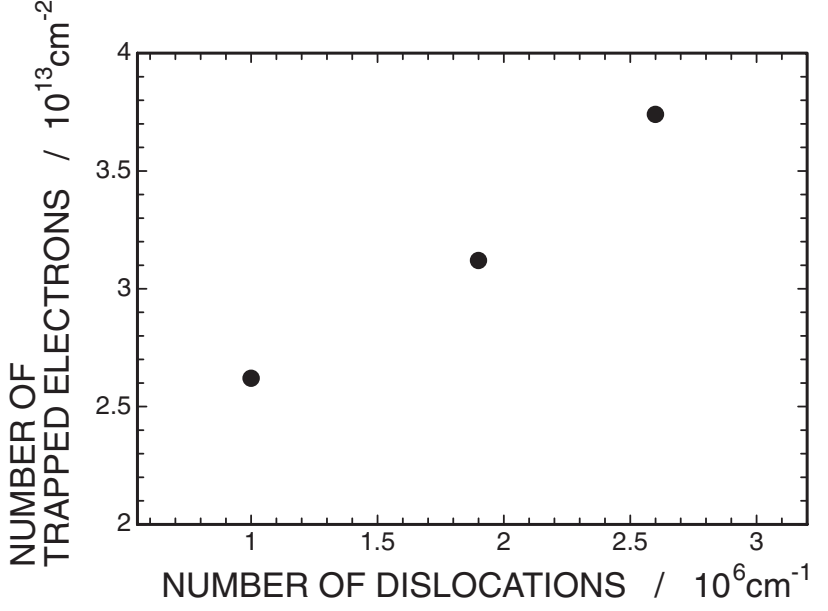

Fig. 10. Excess charges estimated from non-linearity as a function of the number of dislocations obtained from HRTEM images.

because acceptor type dopants form extra electronic states, an interface state, at a deep level in the band gap that are effective to increase the energy height of DSB. However, direct EEL measurement revealed that doped Pr should be a donor ${ }^{24)}$ as shown in this section. Figure 11 shows a HAADF-STEM image of a Pr-doped $\mathrm{ZnO}$ bicrystal. The boundary has $\Sigma 7$ relation with a rotational axis and an angle of [0001] and $21.8^{\circ}$, respectively. As seen in the image, brighter contrast exists periodically along the boundary as partially indicated with arrows. In HAADFSTEM imaging, the intensity of atomic columns is approximately proportional to the average of the square of atomic number, $Z{ }^{21)}$ Comparing $Z$ number of $\mathrm{Zn}$ and $\mathrm{O}$ ions, that of $\mathrm{Pr}$ is very large. Thus, atomic columns including $\operatorname{Pr}$ ions are observed with brighter contrast. The image shown in Fig. 11 clearly shows that doped Pr strongly segregates at the grain boundary and they exist at special atomic columns. Figure 11(c) shows $\operatorname{Pr} \mathrm{M}_{4,5}$-edges taken from the atomic column including Pr. It is noted that the EELS shows a typical profile of $\operatorname{Pr} 3+$. This means that doped $\operatorname{Pr}$ is a donor, considering the valence of the $\mathrm{Zn}$ ion to be $2+$. This is contradiction with the past thought that Pr was an acceptor. By 

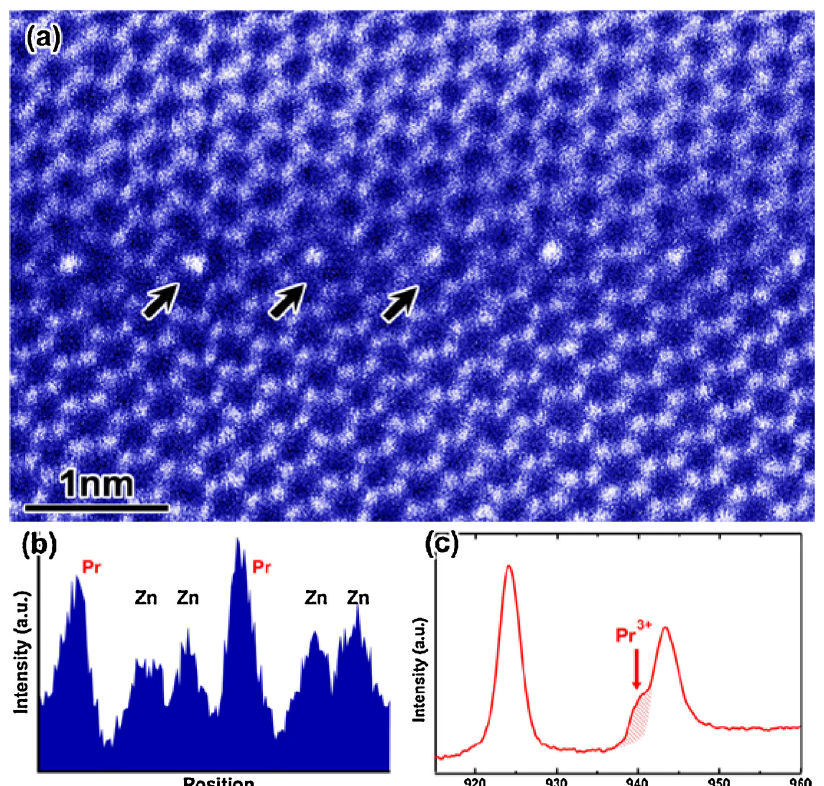

(c)

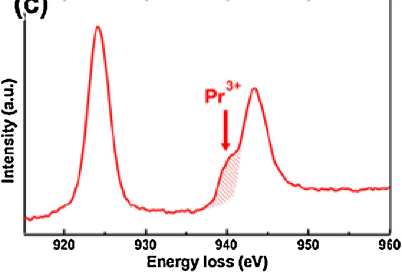

Fig. 11. (Color online) (a) HAADF-STEM image of Pr-doped ZnO $\Sigma 7$ boundary, (b) contrast intensity profile along a boundary, and (c) $\operatorname{Pr~M}_{4,5^{-}}$ edge ELNES around an atomic column including Pr.

carrying out $a b$-initio calculations, the vacancy formation energy of $\mathrm{Zn}$ point defects is found to decrease by the segregation of $\mathrm{Pr}$. The increment of varistic effect due to doping of $\mathrm{Pr}$ is closely related to the accumulation of $\mathrm{Zn}$ vacancies, which are induced by Pr doping. On the other hand, the segregation feature of Pr at $\mathrm{ZnO}$ grain boundaries varies with a grain orientation relationship. Results performed for other types of Pr-doped $\mathrm{ZnO}$ bicrystals are shown in the Ref. 64.

\section{Interface structural change induced by dopant segregation}

There are many cases that mechanical properties are improved by doping in practical materials. One example is the case of cemented carbide, i.e., WC-Co based cemented carbides. ${ }^{65)}$ Cemented carbides are composite materials that are WC hard grains bound by metallic Co. They exhibit high strength and high hardness so that they are often used for mechanical machining tools and wear-resistant materials. In practical use, to increase their mechanical properties, other carbides are often doped as an inhibitor of WC grains. ${ }^{5), 66)} \mathrm{VC}$ is a typical inhibitor that exhibits a strong inhibition effect. ${ }^{5)-7), 67)-69)}$ Figure 12 shows TEM bright filed images taken from undoped and VC-doped $\mathrm{WC}-\mathrm{Co}$ composites. ${ }^{67)}$ As seen in the images, WC grain size is largely decreased by $\mathrm{VC}$ doping. In addition, $\mathrm{VC}$ doping induces an interface structural change as indicated with arrows in the image of (b). The interface at WC/Co clearly exhibits very fine facets. HRTEM observation combined with nano-probe analysis has revealed that doped $\mathrm{VC}$ strongly segregates at $\mathrm{WC} / \mathrm{Co}$ interfaces and they retard step migration on the surface of WC. Figure 13 shows a HRTEM image taken from a VC-doped WCCo composite. ${ }^{67)} \mathrm{WC}$ grains have a rectangular shape with two habits of (0001) and $\{10 \overline{1} 0\}$, which are typical of a hexagonal crystal structure as shown in Fig. 12(a). Also in VC-doped composite, $\mathrm{WC} / \mathrm{Co}$ interfaces consist of the two habits. However, it is noted that the interface changes into a shape like a stair. The habit planes are showing a very fine step structure. Doped V strongly segregates at these fine-steps, which was revealed by

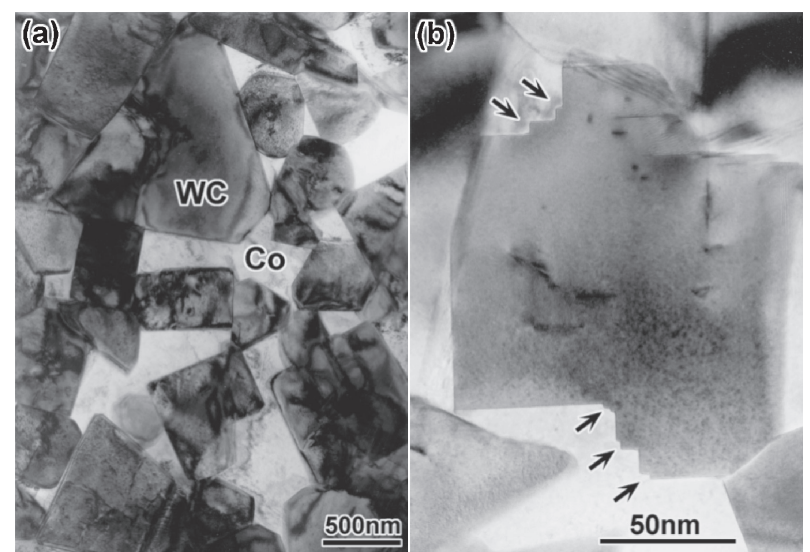

Fig. 12. TEM bright field images of (a) WC-12 mass \% Co and (b) 0.5 mass \%VC-doped WC-12 mass \%Co cemented carbides.

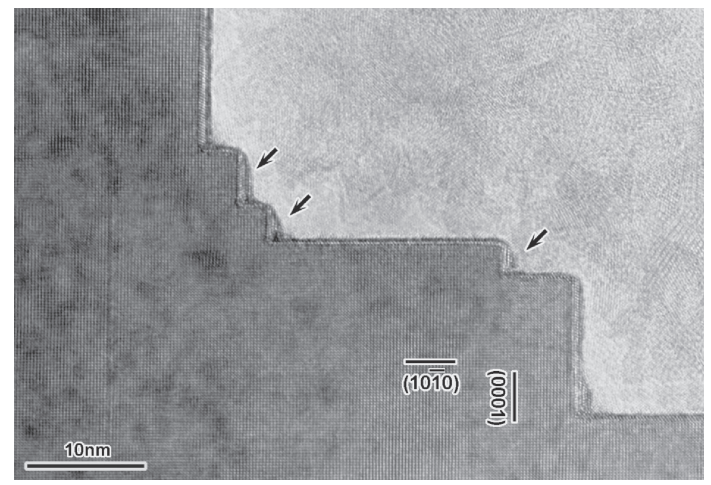

Fig. 13. HRTEM image of WC/Co interface in VC-doped WC-Co composite.

EDS. ${ }^{67)}$ The segregated V strongly retards a step migration of $\mathrm{WC}$, which results in the strong inhibition of WC grain growth. ${ }^{69)}$ Recently the existence of the $(\mathrm{W}, \mathrm{V}) \mathrm{C}_{X}$ layers during liquid-phase sintering is confirmed by $a b$-initio calculations. ${ }^{70)}$

\section{Dislocation nanowire}

In general, dislocations are often thought to decrease physical properties, for example, in emission devices and electron conductive materials. The dislocations are a kind of onedimensional lattice defect including an extra half plane, which gives some lattice expansion at a dislocation core. Thus, some attractive physical properties often appear. For example, the diffusivity is often enhanced at the dislocation core, what we call, pipe-diffusion. With the pipe-diffusion, we can selectively diffuse dopants into a dislocation core. By this technique, we can prepare nano-wires even inside crystals. ${ }^{71), 72)}$ For this purpose, we chose alumina single crystal (sapphire), which is a typical insulating material. At first, a sapphire is mechanically deformed at high temperature. In order to introduce a higher density of dislocations, a two stage deformation technique was performed. Specifically, a sapphire was initially deformed slightly at higher temperature to introduce glide dislocations. After that, it was deformed again at lower temperature. To carry out the two stage deformation, dislocations more than $10^{9} \mathrm{~cm}^{-2}$ was successfully introduced into a sapphire without any fracture. To let the dislocations in vertical alignment in a specimen, a mechanically thinned plate of deformed sapphire was annealed at high temperature. Dislocations inside a deformed sapphire are aligned straight by mirror forces. By evaporating metallic Ti on a 

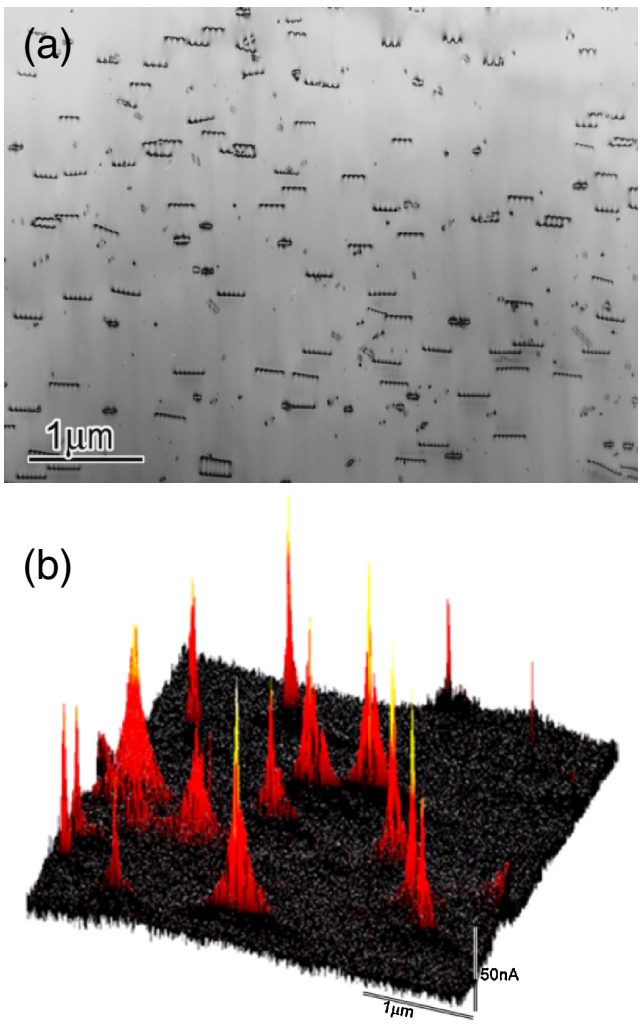

Fig. 14. (Color online) (a) TEM bright field image of unidirectional dislocation structure in a deformed sapphire, and (b) a current image obtained by contact mode AFM.

surface of the treated plates and annealing at higher temperature to enhance pipe-diffusion, evaporated Ti selectively diffuses into dislocation cores. As a result, electrically conductive nano-wires can be obtained as shown in Fig. 14. ${ }^{71)}$ Figure 14(b) is a current map obtained by a contact mode AFM. As we can see, some current spikes can be seen in the map, which shows current flow at Ti-doped dislocation cores. Such nano-wires have also been successfully prepared in GaN and AlN. ${ }^{73), 74)}$

\section{Summary}

In this review, some detailed researches concerning the control of the grain growth in $\mathrm{BaTiO}_{3}$, the electron transport behaviors across single grain boundaries in n-type $\mathrm{BaTiO}_{3}, \mathrm{SrTiO}_{3}$ and $\mathrm{ZnO}$, and the boundary structure change in VC-doped $\mathrm{WC}-\mathrm{Co}$ cemented carbide were summarized. The grain growth, the electron transport and the boundary structures are closely related to atomic configurations at grain boundaries and interfaces, which are induced by a small amount of dopants and point defects. To know the details, we applied nano-scale analysis based on transmission electron microscopy with X-ray dispersive spectroscopy and electron energy-loss spectroscopy. Recent development of transmission electron microscopy, including compositional and electronic analysis techniques, has been outstanding. Especially, Cs-corrected high resolution scanning transmission electron microscope enables us to obtain valuable information even at single atomic columns in a material. To apply such nano/sub-nano analysis technique to practical materials has become essential in the martial science field.

Acknowledgements The author would like to acknowledge the collaboration of Prof. K. Hayashi (Tokyo Inst. Tech.), Dr. M. Demura (NIMS), Prof. K. Matsunaga (Kyoto U.), Prof. F. Oba (Kyoto U.),
Prof. A. Nakamura (Osaka City U.), Prof. Y. Sato (U. Tokyo), Prof. N. Shibata (U. Tokyo), Prof. T. Mizoguchi (U. Tokyo). The author would like to express gratitude to Prof. T. Sakuma (Kochi U. Tech.) and Prof. Y. Ikuhara (U. Tokyo). A part of this study was financially supported by Grant-in-Aid for Scientific Research on Priority Areas "Nano Materials Science for Atomic Scale Modification 474" from Ministry of Education, Culture, Sports, Science and Technology (MEXT) of Japan.

\section{References}

1) Y. Ikuhara, I. Tanaka and T. Yamamoto, Mater. Trans., 50, 939 (2009).

2) Y. Ikuhara, Mater. Integr., 22, 1 (2009).

3) T. K. Gupta, J. Am. Ceram. Soc., 73, 1817-1840 (1990).

4) K. Mukae, K. Tsuda and I. Nagasawa, Jpn. J. Appl. Phys., 16, 1361-1368 (1977).

5) K. Hayashi, Y. Fuke and H. Suzuki, J. Jpn. Soc. Powder Powder Metall., 13, 67-71 (1972).

6) T. Taniuchi, K. Okada and T. Tanase, in: Proceedings of the 14th International Plansee Seminar, Ed. by H. Bildstein and R. Eck, Metallwerk Plansee, Reutte (1993) Vol. 3, pp. 639-645.

7) M. Kawakami, O. Terada and K. Hayashi, J. Jpn. Soc. Powder Powder Metall., 51, 576-585 (2004).

8) W. Heywang, Solid-State Electron., 3, 51-58 (1961).

9) H. Irig, J. Am. Ceram. Soc., 64, 617-620 (1981).

10) H. M. Al-Allak, A. W. Brinkman, G. J. Russell and J. Woods, J. Appl. Phys., 63, 4530-4535 (1988).

11) A. B. Alles and V. L. Burdick, J. Am. Ceram. Soc., 76, 401408 (1993).

12) "Silicon Nitride Ceramics-Scientific and Technological Advances," Ed. by I. W. Chen, P. F. Becher, M. Mitomo, G. Petzow and T.-S. Yen, MRS Proceedings, Mater. Res. Soc. 287, Pittsburgh, Pennsylvania (1993) p. 147.

13) H. Yoshida, Y. Ikuhara and T. Sakuma, Acta Mater, 50, 29552966 (2002)

14) H. Yoshida, S. Hashimoto and T. Yamamoto, Acta Mater., 53, 433-440 (2005).

15) "Transmission electron microscopy," Ed. by D. B. Williams and C. B. Carter, Plenum Press, New York (1996).

16) Y. Ikuhara and T. Yamamoto, Denshi Kenbikyo, 37, 129-133 (2002).

17) Y. Ikuhara and T. Yamamoto, Denshi Kenbikyo, 37, 51-55 (2002).

18) K. Moriyama, K. Shirota and T. Yamamoto, Ceramics, 35, 376-381 (2000).

19) K. Urban, B. Kabius, M. Haider and H. Rose, J. Electron Micros., 48, 821-826 (1999).

20) O. L. Krivanek, N. Dellby and A. R. Lupini, Ultramicroscopy, 78, 1-11 (1999).

21) S. J. Pennycook and D. E. Jesson, Ultramicroscopy, 37, 14-38 (1991).

22) H. Sawada, E. Okunishi, K. Tomita and T. Yamamoto, Kenbikyo, 41, 11-15 (2006).

23) T. Mizoguchi, W. Olovsson, H. Ikeno and I. Tanaka, Micron, 41, 695-709 (2010).

24) Y. Sato, J. P. Buban, T. Mizoguchi, N. Shibata, M. Yodogawa, T. Yamamoto and Y. Ikuhara, Phys. Rev. Lett., 97, 106802 (2006).

25) T. Yamamoto, Y. Ikuhara and T. Sakuma, Sci. Technol. Adv. Mater., 1, 97-104 (2000).

26) S. Lay, J. Thibault and S. Hamar-Thibaul, Philos. Mag., 83, 1175-1190 (2003).

27) N. Shibata, S. J. Pennycook, T. R. Gosnell, G. S. Painter, W. A Shelton and P. F. Becher, Nature, 428, 730-733 (2004).

28) N. Shibata, G. S. Painter, R. L. Satet, M. J. Hoffmann, S. J. Pennycook and P. F. Becher, Phys. Rev. B, 72, 140101(R) (2005).

29) J. P. Buban, K. Matsunaga, J. Chen, N. Shibata, W. Y. Ching, T. Yamamoto and Y. Ikuhara, Science, 311, 212-215 (2006). 
30) D. Hennings, R. Janssen and P. Reynen, J. Am. Ceram. Soc., 70, 23-27 (1987).

31) S.-Y. Choi and S.-J. L. Kang, Acta Mater., 52, 2937-2943 (2004).

32) B. K. Lee, S. Y. Chung and S. L. Kang, Acta Mater., 48, 15751580 (2000).

33) H. Schmeltz and A. Mayer, Ber. Dtsch. Keram. Ges., 59, 436440 (1982).

34) T. Yamamoto, T. Sakuma and P. R. Rios, Scr. Mater., 39, 17131717 (1998).

35) P. R. Rios, T. Yamamoto, T. Kondo and T. Sakuma, Acta Mater., 46, 1617-1623 (1998).

36) R. C. DeVries, J. Am. Ceram. Soc., 42, 547-558 (1959).

37) B. K. Lee, S. Y. Chung and S. J. L. Kang, Acta Mater., 48, 1575-1580 (2000).

38) B. K. Lee and S. J. L. Kang, Acta Mater., 49, 1373-1381 (2001).

39) E. A. Holm and S. M. Foiles, Science, 328, 1138-1141 (2010).

40) T. Yamamoto, Br. Ceram. Trans., 94, 196-200 (1995).

41) T. Yamamoto, K. Hayashi, Y. Ikuhara and T. Sakuma, J. Mater. Res., 13, 3449-3452 (1998).

42) I. Tanaka, I. Nakajima, J. Kawai, H. Adachi, H. Gu and M. Ruhle, Philos. Mag. Lett., 75, 21-27 (1997).

43) T. Yamamoto, Y. Ikuhara, K. Hayashi and T. Sakuma, Key Eng. Mater., 169-170, 113-116 (1999).

44) T. Yamamoto, K. Hayashi, Y. Ikuhara and T. Sakuma, Philos. Mag. Lett., 79, 327-331 (1999).

45) K. Hayashi, T. Yamamoto and T. Sakuma, J. Am. Ceram. Soc., 79, 1669-1672 (1996).

46) T. Yamamoto and T. Sakuma, J. Am. Ceram. Soc., 77, 11071109 (1994).

47) N. Kataoka, K. Hayashi, T. Yamamoto, Y. Sugawara, Y. Ikuhara and T. Sakuma, J. Am. Ceram. Soc., 81, 1961-1963 (1998).

48) P. Gerthsen and B. Hoffmann, Solid-State Electron., 16, $617-$ 622 (1973).

49) H. Nemoto and I. Oda, J. Am. Ceram. Soc., 63, 398-401 (1980).

50) H. Sumino, O. Sakurai, K. Shinozaki and N. Mizutani, J. Ceram. Soc. Japan, 100, 97-100 (1992).

51) H. Ogawa, M. Demura, T. Yamamoto and T. Sakuma, J. Mater. Sci. Lett., 14, 537-538 (1995).

52) M. Kuwabara, K. Morimo and T. Matsunaga, J. Am. Ceram. Soc., 79, 997-1001 (1996).
53) T. Gemming, S. Nufer, W. Kurtz and M. Ruhle, J. Am. Ceram. Soc., 86, 581-589 (2003).

54) E. Tochigi, N. Shibata, A. Nakamura, T. Mizoguchi, T. Yamamoto and Y. Ikuhara, Acta Mater., 58, 208-215 (2010).

55) A. Nakamura, E. Tochigi, N. Shibata, T. Yamamoto and Y. Ikuhara, Mater. Trans., 50, 1008-1014 (2009).

56) N. Shibata, F. Oba, T. Yamamoto and Y. Ikuhara, Philos. Mag., 84, 2381-2415 (2004).

57) T. Yamamoto and Y. Ikuhara, J. Electron Microsc., 50, 485$488(2001)$

58) S.-Y. Choi, J. P. Buban, M. Nishi, H. Kageyama, N. Shibata, T. Yamamoto, S.-J. L. Kang and Y. Ikuhara, J. Mater. Sci., 41, 2621-2625 (2006).

59) D. G. Brandon, B. Ralph, S. Ranganathan and M. S. Wald, Acta Metall., 12, 813-821 (1964).

60) D. G. Brandon, Acta Metall., 14, 1479-1484 (1966).

61) T. Tanaka, K. Matsunaga, Y. Ikuhara and T. Yamamoto, Phys. Rev. B, 68, 205213 (2003).

62) F. Greuter and G. Blatter, Semicond. Sci. Technol., 5, 111-137 (1990).

63) D. R. Clarke, J. Am. Ceram. Soc., 82, 485-502 (1999).

64) Y. Sato, T. Yamamoto and Y. Ikuhara, J. Am. Ceram. Soc., 90, 337-357 (2007).

65) F. V. Lenel, "Powder Metallurgy and Its Applications," Metal Powder Industries Federation, Princeton, NJ (1980).

66) J. Gurland, Trans. Metall. Soc. AIME, 227, 1146-1151 (1963).

67) T. Yamamoto, Y. Ikuhara and T. Sakuma, Sci. Technol. Adv. Mater., 1, 97-104 (2000).

68) S. Lay, J. Thibault and S. Hamar-Thibaul, Philos. Mag., 83, 1175-1190 (2003).

69) M. Christensen and G. Wahnstrom, Int. J. Refract. Met. Hard Mater., 24, 80-88 (2006).

70) S. Johansson and G. Wahnstrom, Phil. Mag. Lett., 90, 599-609 (2010).

71) A. Nakamura, K. Matsunaga, J. Tohma, T. Yamamoto and Y. Ikuhara, Nat. Mater., 2, 453-456 (2003).

72) Y. Ikuhara, Prog. Mater. Sci., 54, 770-791 (2009).

73) Y. Tokumoto, S. Amma, N. Shibata, T. Mizoguchi, K. Edagawa, T. Yamamoto and Y. Ikuhara, J. Appl. Phys., 106, 124307 (2009).

74) S. Amma, Y. Tokumoto, K. Edagawa, N. Shibata, T. Mizoguchi, T. Yamamoto and Y. Ikuhara, Appl. Phys. Lett., 96, 193109 (2010).

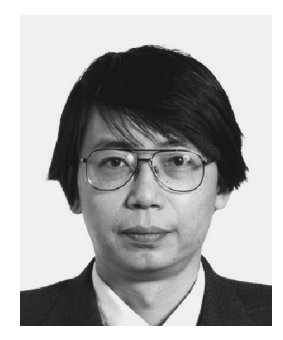

Takahisa Yamamoto is an associate professor at the Department of Advanced Materials Science, the University of Tokyo. He received his B.Eng. in 1987 and M.Eng. in 1989. After receiving his M.Eng., he joined the Department of Materials Science as a research associate. He received his Ph.D. in 1997 with the microstructure control of $\mathrm{BaTiO}_{3}$ sinters and the PTCR effect across single grain boundaries. In 2001, he joined the Department of Advanced Materials Science as an associate professor. His research interests are focused on electrical properties across grain boundaries and interfaces in ceramics. Currently, this includes a control of point defects to develop more smart electroceramic devices by combining material processing and atomistic scale analysis by HRTEM, STEM, EDS, and EELS. 